\title{
GW23-e1874 SIMVASTATIN PREVENT ATHEROSCLEROSIS DEVELOPIMENT THROUGH INHIBITING PROTEIN EXPRESSION OF ADHESION MOLECULES
}

doi:10.1136/heartjnl-2012-302920b.37

Li xiaoyan, Lu ying, Zhang ping, Ou caihong. The Third Affiliated Hospital of Sun Yatsen University

Objectives To explore the effects of simavastatin on protein expressions of intercellular adhesion molecule 1 (ICAM-1), vascular cell adhesion molecule 1 (VCAM-1) and endothelial leukocyte adhesion molecule 1 (E-selectin) of cholesterol diet rabbits and the underlying mechanisms of the beneficial effects of simvastatin on atherosclerosis (AS).

Methods Twenty-four male rabbits were randomly divided into three groups: normal diet groups, cholesterol diet groups and the simvastatin groups which received both cholesterol diet and simastatin $5 \mathrm{mg} / \mathrm{kg} / \mathrm{d}$ intragastrically. ELISA was used to detect plasma protein levels of ICAM-1, VCAM-1 and E-selectin of rabbits at 0, 8 and 16 weeks, respectively. After 16 weeks, rabbits were executed and the artery wall was harvested for the pathologic and morphologic observations. Western blot and RT-PCR were used to 
determine ICAM-1, VCAM-1 and E-selectin level of rabbits artery wall.

Results Compared with normal diet groups, the expression of ICAM-1, VCAM-1 and E-selectin in cholesterol diet groups plasma and artery wall was obviously increased both in protein and mRNA level. $(n=6 ; p<0.01)$. However, simavastatin could inhibit the formation of atherosclerotic plaques obviously; suppress plasma and artery wall protein expression and mRNA level of ICAM-1, VCAM-1 and E-selectin $(n=6 ; p<0.01)$.

Conclusions Simvastatin could ameliorates atherosclerosis (AS) through inhibiting expression level of plasma and artery wall adhesion molecule. 